\section{Ethics of Surgical Complications}

\author{
Simisade Adedeji · Daniel K. Sokol · \\ Thomas Palser · Martin McKneally
}

(C) Société Internationale de Chirurgie 2009

\section{Defining surgical complications}

Whatever the place or period, surgical complications have been an inevitable part of surgical practice. It is not surprising, then, that studies on various aspects of surgical complications are plentiful. A PubMed search returned nearly 800 articles with the phrase "surgical complication" and its plural form in the title. However, despite the importance and prevalence of the matter, there is at present no agreed definition of a surgical complication [1].

Published definitions of the term range from the straightforward ("any undesirable result of surgery") to the more elaborate $[2,3]$. In this article, we propose to use the following definition, which, although unwieldy, captures more accurately the attributes of a surgical complication.

\footnotetext{
S. Adedeji

Department of Primary Care and Social Medicine, Imperial College London, St. Dunstan's Road, London W6 8RP, UK

e-mail: simisade.adedeji@imperial.ac.uk;

simisade@doctors.org.uk

D. K. Sokol ( $\square)$

St. George's, University of London, Cranmer Terrace, London SW17 ORE, UK

e-mail: daniel.sokol@talk21.com

T. Palser

Clinical Effectiveness Unit, Royal College of Surgeons of England, Lincoln's Inn Fields, London WC2A 3PE, UK

M. McKneally

Department of Surgery and Joint Centre for Bioethics, University of Toronto, 88 College Street, Toronto,

ON M5G 1L4, Canada
}

A surgical complication is any undesirable, unintended, and direct result of surgery affecting the patient which would not have occurred had the surgery gone as well as could reasonably be hoped [4].

The purpose of this overview is to delineate the key ethical issues relating to surgical complications. As space is limited and the scope of the subject vast, we consider the issues from the surgeon's perspective rather than that of other members of the surgical team.

\section{Four principles of medical ethics: a dominant moral framework}

During the 1970s, the American philosophers Tom Beauchamp and James Childress introduced the highly influential "Four Principles" approach to medical ethics [5]. Their conceptual framework relies on the application of four basic moral principles that, alone or in combination, help identify and resolve ethical issues in medicine.

The principles are neither hierarchically ordered nor invariably binding. They are "prima facie," meaning that they are binding unless they conflict with another principle. When this occurs, the individual must balance the competing moral obligations against each other and, through sound judgement, determine which is most morally compelling. Hence, a surgeon may override the prima facie obligation to obtain informed consent if emergency surgery is needed to save the life of a barely conscious polytrauma patient. A brief description of the principles follows:

1. Respect for autonomy Literally meaning self rule, autonomy usually refers to people's ability to make choices based on their own beliefs and values. In health care, respect for autonomy requires doctors to 
respect a competent patient's deliberated wishes and provide adequate information to support patients in their decision-making.

2. Beneficence This refers to the Hippocratic commitment to benefit patients by acting in their best interests. Because the conception of what constitutes benefit (and harm) varies from person to person, this principle usually requires respecting patient autonomy.

3. Nonmaleficence This principle refers to the moral obligation not to cause harm to patients. Surgery, with its necessary incision, could be seen to defy this principle. Indeed, all attempts to benefit patients, whether through words, drugs, or procedures, carry risks of harm. Hence nonmaleficence is best described as the obligation to avoid causing net harm to patients and should be considered in conjunction with the principle of beneficence.

4. Justice In health care ethics, the principle of justice primarily refers to the obligation to distribute scarce health care resources fairly. It also includes the obligation to respect people's human rights and to respect morally acceptable laws.

These four broad principles generate more specific rules. Under respect for autonomy, for example, are rules such as "obtain consent," "respect confidentiality," and "avoid deception." Although the "principlist" approach does not claim to single-handedly resolve all ethical problems (because judgement is also needed), it provides a useful checklist of basic moral considerations with which to examine ethical issues. For our purposes, the four principles serve as a framework to consider systematically the ethics of surgical complications.

Table 1 is a principlist typology of ethical issues in surgery. Some of the ethical issues, such as the duty to possess adequate technical skill, are captured by more than one principle. We then elaborate on those issues pertaining more specifically to surgical complications. As will become apparent, there is a significant overlap between the ethics of surgery and the ethics of surgical complications. This is not surprising given that surgical complications fall within the realm of surgical practice and that the most efficient way of reducing complications is to strive for high standards of surgical care.

\section{Application of the four principles approach to surgical complications}

\section{Respect for autonomy}

The main autonomy-related issue in surgical ethics is the prima facie duty to obtain adequately informed consent.
Table 1 Typology of ethical issues in surgery

\begin{tabular}{|c|c|}
\hline Principle & Ethical issues in surgery \\
\hline $\begin{array}{l}\text { Respect for } \\
\text { autonomy }\end{array}$ & $\begin{array}{l}\text { Informed consent for surgery } \\
\text { Truth-telling (to patients, relatives, and colleagues) } \\
\text { Consent for the involvement of trainees in surgical } \\
\text { procedures } \\
\text { Confidentiality } \\
\text { Respecting patient's requests (for procedures/ } \\
\text { particular surgeons) } \\
\text { Good communication skills }\end{array}$ \\
\hline Beneficence & $\begin{array}{l}\text { Surgical competence } \\
\text { Ability to exercise sound judgment } \\
\text { Continuous professional development } \\
\text { Research and innovation in surgery } \\
\text { Responsible conduct } \\
\text { Functioning equipment and optimal operating } \\
\text { conditions } \\
\text { Minimizing harm (including pain control) } \\
\text { Good communication skills }\end{array}$ \\
\hline Nonmaleficence & $\begin{array}{l}\text { Surgical competence } \\
\text { Continuous professional development } \\
\text { Ability to exercise sound judgment } \\
\text { Recognizing the limits of one's professional } \\
\text { competence } \\
\text { Research and auditing } \\
\text { Disclosure and discussion of surgical } \\
\text { complications including medical errors } \\
\text { Good communication skills }\end{array}$ \\
\hline Justice & $\begin{array}{l}\text { Allocation of scarce resources } \\
\text { Legal issues } \\
\text { Respecting human rights } \\
\text { Whistle blowing }\end{array}$ \\
\hline
\end{tabular}

Informed consent is all the more important in surgery given the invasiveness of the act, the short-term harm, and the temporarily unconscious state of the patient during the procedure.

One difficulty relates to the degree of disclosure necessary to satisfy the consent conditions. How much information is adequate? It is neither practical nor desirable to list all possible complications to a patient. Not only would such a disclosure be time-consuming, but providing too much information can reduce rather than enhance a patient's autonomy by confusing the patient. Furthermore, excessive information about theoretical complications may frighten the patient into refusing operations that may in reality be relatively low risk and highly beneficial. Clear disclosure that is neither deficient nor excessive in detail and that is tailored to the patient's own situation requires a combination of good judgment and communicative ability. 
Three factors affect the extent of the disclosure: the severity of the complication (the more serious the complication, the more inclined the surgeon should be to divulge it), the likelihood of the complication to occur (again, the more likely the complication, the greater the need to disclose it to the patient prior to surgery), and the patient's information preferences. Ideally, the surgeon tailors the information to the needs and circumstances of the individual patient. For example, a surgeon should be particularly thorough when discussing the possible complications of removing vocal cord nodules with a professional singer or hand surgery with a sleight-of-hand magician.

Some courts in England have applied the "reasonable person standard" to determine how much information should be given [6]. This standard requires the surgeon to provide information that a reasonable person in the patient's circumstances would want to know. However, what constitutes a reasonable patient is not an objective reality and, once again, to some extent must rely on the surgeon's judgment.

In rare cases, a patient may decline all information about the surgery or surgical complications and delegate the decision-making to the surgeon. The trade union for doctors and medical students in the United Kingdom and also the governing body of doctors give clear guidance regarding this point: The surgeon must give at least basic information about the procedure. What counts as basic information depends on the particular circumstances, the severity of the condition, and the procedure's risks [7].

What if a patient requires an unanticipated procedure intraoperatively, and explicit consent has not been obtained? For example, a surgeon may be confronted intraoperatively with a large renal cancer during the course of a colectomy. The patient may now require a more extensive procedure. The decision to perform the new procedure there and then depends on a combined evaluation of severity and urgency. Whatever the decision taken, the surgeon must be prepared to justify his or her decision to the courts or the General Medical Council. It is thus wise, whenever practicable, to ask the advice of an experienced colleague. Some complications, however, require immediate action even in the absence of consent. Hence, a surgeon may need to perform an emergency hysterectomy to control massive bleeding in an obstetric patient with complicated placenta praevia.

As patients cannot be informed of all possible eventualities, it may be advisable to seek more general consent to treat any problems that may arise during the surgery. Another possible solution, which could be used in conjunction with broadening the scope of the consent, is to offer patients the possibility of appointing a proxy (or, in the United Kingdom, a Lasting Power of Attorney) to act on the patient's behalf should an unforeseen situation arise during surgery.

\section{Disclosing complications}

It may be tempting for a surgeon convinced that surgery is best for the patient to ignore or downplay the severity or likelihood of a surgical complication. Mentioning frightening facts may, after all, deter the patient. Such a paternalistic approach is today widely condemned, at least in the Western context, and has been replaced by a strong concern for truth-telling and openness.

On a similar issue, a surgeon who has made a mistake may attempt to disguise it by calling the error a surgical complication, thereby relying on the patient's inference that this eventuality was inevitable. This is deceptive and paternalistic. The duty of honesty requires the surgeon, in all but the most exceptional circumstances where serious harm may realistically result, to specify whether the complication was caused by an error. This requirement also falls within the principle of justice as the patient has a right to this information. The patient may be entitled to compensation.

\section{Trainees}

More experienced surgeons are likely to have lower complication rates than less experienced surgeons [8]. Should patients who demand to have their operation performed by a consultant, rather than a junior registrar, have their requests granted on the basis of respecting autonomy and the greater likelihood of benefit? When challenged, the patient may ask the consultant: "Who would you want to operate on your child?"

It could be argued that the principle of beneficence requires the most experienced surgeon, or at least the one with the best track record, to perform the procedure. This should reduce the likelihood of surgical complications. Aside from the impracticality of this position, the principle of beneficence applies to other patients and is tempered by the principle of justice. One patient may ask a consultant surgeon to repair his inguinal hernia, but at the same time another patient may require a splenectomy. The surgeon has a duty of beneficence to both patients and, if unable to do both, must decide which patient to operate on. Which theory of justice should be used to resolve this moral conundrum?

The consultant may decide to perform the more complex and higher-risk splenectomy and delegate the relatively straightforward hernia repair to the registrar. This is an example of distributive justice. The experienced surgeon is, by definition, a limited resource that should be shared fairly among those in medical need. Respect for patient 
autonomy, although requiring surgeons to respect autonomous refusals of treatment, does not necessarily require them to respect positive requests (e.g., "I demand that Professor Smith perform my appendectomy!") for treatment or for a particular surgeon.

The right to health care surely entitles patients to adequate health care, rather than "best care." By definition, few people and institutions can provide the best care. Moreover, acceding to patients' requests for the best care would have negative consequences on the training of less experienced surgeons and their ability to reduce the occurrence of surgical complications. The duty of beneficence is not limited to current patients but extends to future patients, and they too should benefit from high standards of surgery. This can occur only if more junior surgeons are allowed to hone their surgical skills under appropriate supervision.

On occasion, during the process of obtaining consent, a surgeon confirms to the patient that he or she will be performing the operation, even though parts of the operation are performed by a junior surgeon. Should patients be informed that their operation, in whole or in part, may be performed by a junior surgeon? We see no good justification for not informing patients, at the time of obtaining consent, that the senior surgeon will be assisted by a trainee. Disclosing this additional piece of information is not time-consuming, and patients are unlikely to protest, especially if reassured that the trainee will be under constant supervision and that this is common practice. Withholding information about the involvement of the trainee is a form of deception by omission, particularly if the patient is led to believe that the senior surgeon alone will perform the operation.

\section{Beneficence}

To benefit their patients and reduce the likelihood of complications, surgeons must be competent and knowledgeable. This encompasses both surgical technique and the ability to recognize the limits of their professional competence. Not overestimating one's abilities requires the surgeon to possess the virtue of humility, broadly defined as an awareness of one's strengths and, especially, one's weaknesses. In cases deemed beyond their expertise, surgeons should refer the patient to specialist colleagues.

Good surgical judgement is also necessary to determine the appropriateness of surgery based on an evaluation of the risks, burdens, and benefits of surgery for that particular patient. As such an assessment is to some extent subjective, the surgeon should generally make this decision with the individual patient, whose beliefs and values will shape the final decision. Such wisdom of judgment, by reducing the likelihood of harm, also falls under the principle of nonmaleficence.
The appeal to humility and wisdom shows that the four principles approach can be enriched by an account of virtues. Unlike principles, which focus on action, virtues concern an agent's moral character. Moral action requires both the possession of virtues and knowledge of moral principles because in order to discharge the duties required by the principles a surgeon must exercise discernment, integrity, benevolence, trustworthiness, humility, conscientiousness, and other virtues.

A conscientious surgeon approaching retirement age may acknowledge that his or her eyesight is failing and that minute vascular work is proving far tougher than previously. Surgeons have a duty to stop operating when their health leads to substandard care. The incidence of surgical complications due to human error is likely to increase with lack of sleep, poor concentration, stress, and other physical and psychological deficiencies. The Senate of Surgery of Great Britain and Ireland (now called the Surgical Forum) advises that surgeons should "avoid lifestyles and habits giving rise to potential risk to patients" [9]. The conscientious surgeon should ensure that the team has adequate rest between shifts and follows appropriate infection control measures to reduce the incidence of iatrogenic complications.

Surgeons rely on technology, from diathermy to the operating room lights. The conscientious surgeon should thus ensure that the equipment is functioning and reliable. In the current climate of limited National Health Service resources, there can be a real pressure to "manage" with suboptimal equipment especially if the fault is discovered intraoperatively. Faulty equipment compromises patient care and increases the likelihood of surgical complications. One of the authors knows of a fatal cesarean section during which the surgeon struggled to control massive hemorrhaging partly as a result of inadequate operating room lights. This problem could have been avoided had the surgeon insisted on better lights instead of believing that he could overcome the deficiency through surgical skill. Wisdom and humility would undoubtedly have helped.

Still under the principle of beneficence, surgeons have a duty to update their knowledge by attending conferences, reading journals, and keeping informed on the latest therapeutic developments in their field. This practice allows patients to benefit from any new or improved surgical techniques that may reduce complication rates. Regrettably, attending courses and meetings is increasingly difficult at a time of shortened study leave and reduced budgets.

The surgical profession as a whole has an obligation to improve surgical outcomes and reduce complications by conducting research and participating in systematic programs of quality improvement. This requires keeping detailed and accurate data on the incidence of adverse events. On occasion, a surgeon may decide to deviate from 
standard protocol and use an innovative technique. As protocols are designed to cut down the risks of complications, the surgeon should be prepared to justify his or her decision to professional colleagues. The burden of proof lies with the innovating surgeon. The Surgical Forum advises that "such innovation is acceptable to the degree that a responsible body of medical opinion would agree that it has been employed in a situation where there was no alternative" [9].

Many of the qualities mentioned above rely on the presence of what Sir William Osler called the "quality of thoroughness" [10]. Thoroughness and meticulousness can be viewed as aspects of the virtue of conscientiousness that are especially pertinent to the ethical surgeon. A high measure of thoroughness in the operating theater and in the surgeon's professional and educational development is necessary for ensuring the highest standards of patient care and the lowest rates of complications.

\section{Nonmaleficence}

The ethical issues discussed under the principle of beneficence-technical ability, recognition of the limits of one's competence, the ability to exercise good judgment, adequate self-care, strict infection control measures, mechanical checks of systems and equipment, and the need for research, quality improvement, and continuous professional development-all contribute to reducing the occurrence of surgical complications and hence also fall under the principle of nonmaleficence.

Good communication is another issue captured by several principles. Poor communication, both in the preoperative conversation about risks and benefits and postoperatively when complications have arisen, can lead to considerable and avoidable emotional distress. Surgeons should communicate appropriately not only to patients but also to the other members of the team, from the anesthetist to the operating theater assistant. Readers no doubt have encountered surgeons whose verbal interactions with team members have been inappropriately rude. Aside from the disrespect this shows toward the victim, safe surgery requires good teamwork; and fear, frustration, or anger can lead individuals to make errors. Substandard communication (however respectfully delivered), such as unclear or insufficiently detailed instructions, can also lead to misunderstandings and reductions in efficiency among the surgical team.

When surgical complications occur, surgeons have a moral obligation to minimize, inasmuch as possible, recurrence of similar complications. Regular "mortality and morbidity" meetings in which surgical teams review any recent complications is one way surgeons fulfill this obligation. Discussions regarding complications and critical analysis of management options, relevant systems, and operating techniques can then follow. Self-reporting and frank discussion nonetheless occur only in an environment propitious to the honest and accurate representation of surgical complications and their cause. A climate of blame is unlikely to lead to open discussion and fruitful improvements. Surgeons may also wish to describe their experiences of surgical complications in journal articles and conferences so others may benefit from any lessons learned.

Justice

As patients have a right to adequate standards of surgical care, surgeons who believe they are putting patients at undue risk of surgical complications must address the problem. This may mean further training to raise their standards or removing themselves from certain cases. If a surgeon recognizes a problem in colleagues that may put patients at undue risk, such as alcoholism or drug dependence, he or she is also under a moral duty to act on this observation, whether by approaching the colleague in difficulty, his or her seniors, or in serious cases a higher authority such as a professional body. A decision not to intervene remains an active decision and is not immune to moral blame.

Another justice-related reason to reduce the occurrence of surgical complications is that such outcomes tend to necessitate further operating time and longer hospital stays for patients. This translates to an economic burden and, in a universal system such as the National Health Service, saps funds from other areas. It also leads to longer waiting times for other patients.

Finally, patients may be entitled to legal remedy and financial compensation in the event of negligent surgical complications.

\section{Conclusion}

Surgeons live and act within a moral community. Although the clinical and technical features of their work are crucially important, so too are the more subtle moral dimensions.

This overview of the ethics of surgical complications has been mostly about moral perception-about identifying the existing issues and showing their plurality and variety-and a little about moral reasoning, explaining why surgeons have duties toward patients using the four principles, enriched by virtues, as a basic moral framework. We hope others will be inspired to engage in these issues in greater depth and in so doing raise the ethical standards of surgery even higher. 
Acknowledgments An early version of this article was delivered at the 1st Advanced Course on Surgical Complications in London, UK, on 22 October 2007. The authors thank the delegates for their helpful questions and comments on the article.

\section{References}

1. Rampersaud Y, Moro ER, Neary MA et al (2006) Intraoperative adverse events and related postoperative complications in spine surgery: implications for enhancing patient safety founded on evidence-based protocols. Spine 31:1503-1510

2. Liposuction.com http://www.liposuction.com/safety. Accessed August 28, 2007

3. Veen M, Lardenoye J, Kastelein GW et al (1999) Recording and classification of complications in a surgical practice. Eur J Surg 165:421-424

4. Sokol D, Wilson J (2008) What is a surgical complication? World J Surg 32:942-944
5. Beauchamp T, Childress J (2001) Principles of biomedical ethics, 5 th edn. Oxford University Press, New York

6. Department of Health (2001) Good practice in consent implementation guide: consent to examination and treatment. DoH Publications, London

7. British Medical Association (2002) Medical ethics today. BMJ Publishing Group, London

8. Faraj A, Drakau N (2007) Press-fit hemiarthroplasty for elderly with femoral neck fracture: high complication rates in operations performed by younger surgeons. Eur J Orthop Surg Traumatol 17:267-271

9. Senate of Surgery of Great Britain, Ireland (1997) Senate paper 2. The surgeon's duty of care: guidance for surgeons on ethical and legal issues. Senate of Surgery of Great Britain and Ireland, London

10. Osler W (1928) Teacher and student. In: Aequanimitas with other addresses to medical students, nurses and practitioners of medicine. Blakiston, Philadelphia, pp 21-43 